This is the final peer-reviewed accepted manuscript of:

A Quantum Chemical Interpretation of Two-Dimensional Electronic Spectroscopy of Light-Harvesting Complexes

Francesco Segatta, Lorenzo Cupellini, Sandro Jurinovich, Shaul Mukamel, Maurizio Dapor, Simone Taioli, Marco Garavelli, and Benedetta Mennucci

Journal of the American Chemical Society 2017139 (22), 7558-7567

The final published version is available online at : http://dx.doi.org/10.1021/jacs.7b02130

Rights / License:

The terms and conditions for the reuse of this version of the manuscript are specified in the publishing policy. For all terms of use and more information see the publisher's website.

This item was downloaded from IRIS Università di Bologna (https://cris.unibo.it/)

When citing, please refer to the published version. 


\title{
A Quantum Chemical Interpretation of Two-Dimensional Electronic Spectroscopy of Light-Harvesting Complexes
}

\author{
Francesco Segatta, ${ }^{\dagger, \ddagger}$ Lorenzo Cupellini, " Sandro Jurinovich, " Shaul Mukamel,,$\S$ \\ Maurizio Dapor, ${ }^{\dagger}$ Simone Taioli, ${ }^{\dagger}, \|$ Marco Garavelli, ${ }^{*, \dagger}$ and Benedetta Mennucci ${ }^{*,}$ \\ European Center for Theoretical Studies in Nuclear Physics and Related Areas \\ (ECT*-FBK) and Trento Institute for Fundamental Physics and Applications \\ (TIFPA-INFN), 38123 Trento, Italy, Dipartimento di Chimica Industriale "Toso \\ Montanari", University of Bologna, Viale del Risorgimento, 4, 40136 Bologna, Italy, \\ Dipartimento di Chimica e Chimica Industriale, University of Pisa, via G. Moruzzi 13, \\ 56124, Pisa, Italy, Department of Chemistry, University of California, Irvine, California \\ 92697-2025, USA, and Faculty of Mathematics and Physics, Charles University, Prague, \\ Czech Republic
}

E-mail: marco.garavelli@unibo.it; benedetta.mennucci@unipi.it

${ }^{*}$ To whom correspondence should be addressed

$\dagger$ European Center for Theoretical Studies in Nuclear Physics and Related Areas (ECT*-FBK) and Trento Institute for Fundamental Physics and Applications (TIFPA-INFN), 38123 Trento, Italy

‡Dipartimento di Chimica Industriale "Toso Montanari", University of Bologna, Viale del Risorgimento, 4, 40136 Bologna, Italy

"Dipartimento di Chimica e Chimica Industriale, University of Pisa, via G. Moruzzi 13, 56124, Pisa, Italy

${ }^{\S}$ Department of Chemistry, University of California, Irvine, California 92697-2025, USA

"Faculty of Mathematics and Physics, Charles University, Prague, Czech Republic 


\begin{abstract}
Nonlinear electronic spectroscopies represent one of the most powerful techniques to study complex multichromophoric architectures. For these systems, in fact, linear spectra are too congested to be used to disentangle the many coupled vibro-electronic processes which are activated. By using a 2D approach, instead, a clear picture can be achieved but only when the recorded spectra are combined to a proper interpretative model. So far, this has been achieved through parameterized Hamiltonians that necessarily introduce biases and/or arbitrary assumptions. In this study, a first principles approach is presented, that combines accurate quantum chemical descriptions with state-of-the-art models for the environment through the use of atomistic and polarizable embeddings. Slow and fast bath dynamics, along with exciton transport between the pigments, are also included. This approach is applied to the 2DES spectroscopy of the Light-Harvesting 2 (LH2) complex of purple bacteria. Simulations are extended over the whole visible-near infrared spectral region to cover both carotenoid and bacteriochlorophyll signals. The obtained results provide an accurate description of excitonic properties and relaxation pathways, and give an unprecedented insight in the interpretation of the spectral signatures of the measured 2D maps.
\end{abstract}

\section{Introduction}

Advances in electronic spectroscopy with femtosecond time resolution, such as the Two Dimensional Electronic Spectroscopy (2DES), have provided new insight into the energy transfer processes in the light-harvesting (LH) pigment-protein complexes present in photosynthetic organisms. ? ? ? The high complexity of these systems, where multiple interacting pigments are embedded in a protein matrix, leads in fact to extremely intricate energy landscapes due to the simultaneous presence of excitonic, vibronic and, eventually, chargetransfer states. By spreading signals along two frequency axes and following the dynamics of excitation with very high time resolution, the 2DES technique allows one to decongest the overlapping transient spectra. As a result, a high density of information is obtained, but to objectively disentangle all these features and reach a detailed and reliable map of the energy transfer network is still a challenge. A possible strategy to solve the latter issue is to integrate the measurements with theoretical models: this has been successfully done by combining data extracted from experiments, and tunable parameters. ? ?

Here we present an accurate quantumchemistry based multiscale approach to simulate 2DES spectra of multichromophoric systems in complex environments for a direct comparison with experiments. By combining two different quantum mechanical descriptions (Density Functional Theory and multireference perturbative RASSCF/RASPT2) with two classical embedding schemes (electrostatic and polarizable) to account for the presence of the environment, we calculate all the quantities needed to build up the exciton-phonon model with a unified theoretical framework able to incorporate slow and fast bath dynamics, along with exciton transport between the pigments. Because of its "ab-initio" character, our approach represents a unique strategy to achieve a clear interpretation of the spectra even when many different pigments are involved and a large range of wavelengths has to be explored. In fact, by switching on and off selected interactions among pigments, a detailed molecular-level analysis of the intricate network of couplings between the multiple electronic processes and the intra/inter-molecular nuclear degrees of freedom can be achieved.

Attempts at calculating $a b$ initio the 2DES spectra of LH systems were conducted on the $\mathrm{LH}_{2}$ ? and $\mathrm{FMO}^{\text {? }}$ complexes. We demonstrate the potential of our approach by simulating the 2DES spectroscopy at low $(77 \mathrm{~K})$ and room temperature of one of the most challenging system, namely the main LH complex of purple bacteria, the Light-Harvesting 2 (LH2) of Rps. Acidophila. Since the determination of its high-resolution structural model reported 
in 1995 by Cogdell and co-workers? , LH2 has in fact been largely studied with different experimental techniques (see Ref. ? for a recent exhaustive review). Many theoretical studies have also been performed;? ? ? ? ? ? ? ? ? however, the accurate interpretation of the excitonic properties of this highly symmetric system is still disputed, mostly owing to the different coupling regimes, which are active on the same time.

The LH2 complex is characterized by a cylindrical $\mathrm{C}_{9}$ structure, containing 27 bacteriochlorophylls $a$ (BChl) and 9 carotenoids (Car) as absorbing pigments. ? The BChls are organized in two rings, a closely packed ring (B850) containing $18 \mathrm{BChls}$ whose molecular plane is perpendicular to the cylinder radius, and a more loosely packed ring (B800) that contains 9 BChls, whose molecular plane is perpendicular to the $\mathrm{C}_{9}$ axis (See Figure ??). The backbone of the Car molecules (Rhodopin Glucoside) encompasses the whole polypeptide chain and bridges the two $\mathrm{BChl}$ rings.

Due to this unique structural arrangement, the bright $\mathrm{Q}_{\mathrm{y}}$ excitations of the B850 BChls are strongly coupled, whereas those of the B800 BChls are more weakly coupled; the coupling between the two rings is also not negligible. This is reflected in the near IR characteristic signal arising from delocalized $\mathrm{Q}_{\mathrm{y}}$ excitons. When we move to the region where the $\mathrm{Q}_{\mathrm{x}}$ transition of the BChls and/or the bright state of the carotenoids are excited, the couplings decrease significantly in both rings as well as between the BChls rings and the connecting Cars.

For the first time, the simulation is here extended over the whole Car- $Q_{x}$ plus $Q_{y}$ visiblenear infrared (VIS-NIR) region. Due to the very different coupling regimes, the exciton model is combined with Redfield and Modified Redfield theories? ? ? ? to describe exciton relaxation within delocalized states of the $\mathrm{Q}_{\mathrm{y}}$ region, and the Förster theory within the Car- $\mathrm{Q}_{\mathrm{x}}$ region.

The obtained results are compared with recently published experimental 2DES data, ? both showing the importance of previously unaddressed aspects of the modeling and revealing new aspects of the recorded signals.
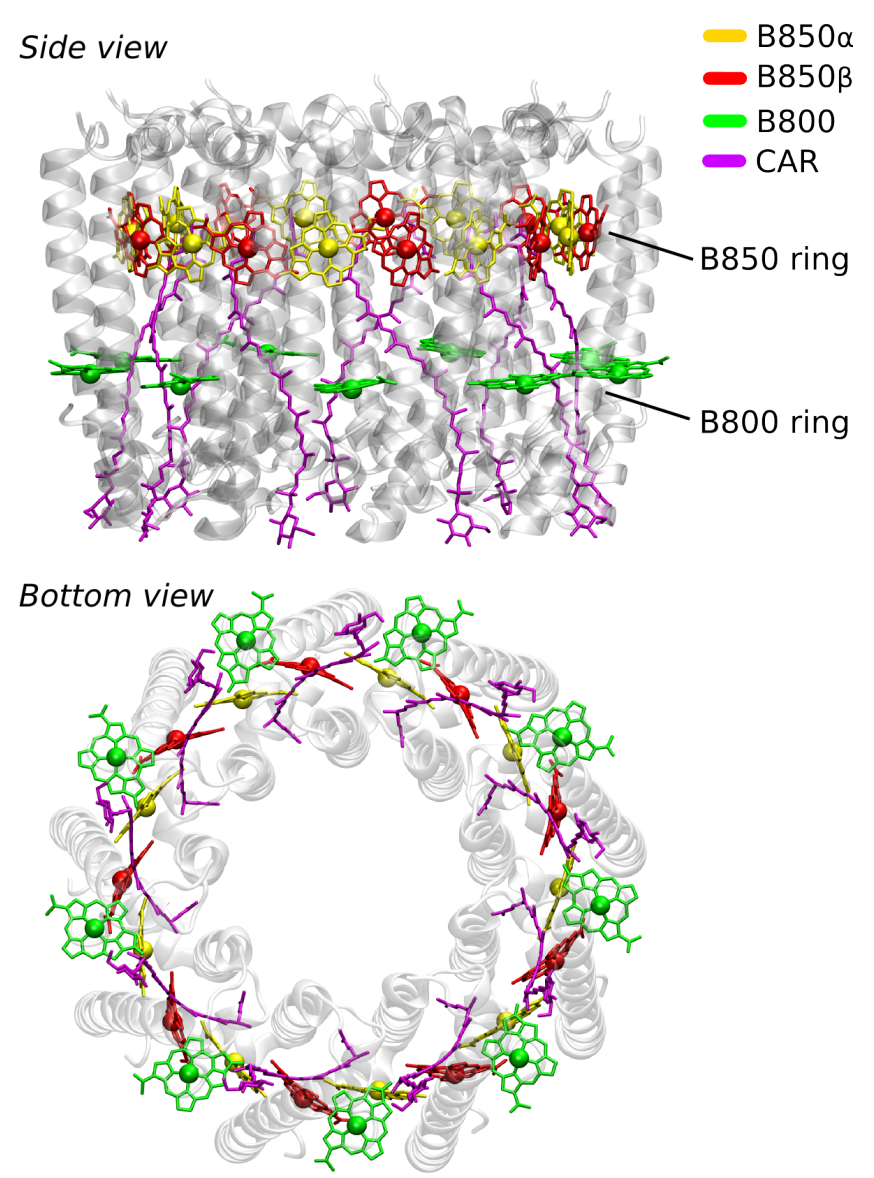

Figure 1: Molecular structure of LH2. Pigments are highlighted using the following color code: yellow for BChl B850 $\alpha$, red for B $850 \beta$, green for B800, purple for the Carotenoid.

Focusing on the $\mathrm{Q}_{\mathrm{y}}$ region, the good agreement between experimental and theoretical maps, obtained when the Coulomb interaction of the full transition densities and the polarization effects of the environment are included, points out the importance of a correct evaluation of the intra and inter-ring couplings. Moreover, we show that the simulations must include the proper shape of excitation and detection pulses if a quantitative agreement of the intensities of B800 and B850 bands in simulated and experimentally recorded 2D maps has to be achieved.

Moving to the Car- $\mathrm{Q}_{\mathrm{x}}$ region of the $2 \mathrm{D}$ map, the calculations clearly identify the signals due to the combined effect of the vibronic progression of the Car's bright $S_{2}$ state and BChl's $\mathrm{Q}_{\mathrm{x}}$ transition, respectively. This is particularly important for those off-diagonal peaks that were 
used to reveal the presence of a previously postulated carotenoid dark state of the same symmetry of $S_{2},{ }^{?}$ which lays in between $S_{2}$ and $\mathrm{Q}_{\mathrm{x}}$ states. The comparison between simulated and measured spectra reveals which signals are due to vibronic progression of BChl's $Q_{x}$ which can be labeled as pure vibrational progressions of the bright Car $S_{2}$ state, and which should indeed be recognized as Car signatures coming from states different than $S_{2}$.

\section{Structure and Methods}

We used the crystal structure of LH2 (PDB code: $1 \mathrm{NKZ}$ ) resolved at $2.0 \AA^{?}$. To ensure a $\mathrm{C}_{9}$ symmetry, we considered only one monomer, which was replicated over the symmetry axis. All details regarding the structure preparation are reported in Section S1 of the Supporting Information.

The multichromophoric system is described in terms of an exciton Hamiltonian as follows:

$$
\hat{H}=\sum_{i} \epsilon_{i}|i\rangle\left\langle i\left|+\sum_{i j} V_{i j}\right| i\right\rangle\langle j|
$$

where $\epsilon_{i}$ is the site energy of the $i$-th chromophore, and $V_{i j}$ is the electronic coupling between chromophores $i$ and $j$. We considered separate Hamiltonians for (i) the near-infrared region of the spectrum, which is determined by only the $\mathrm{Q}_{\mathrm{y}}$ transition of $\mathrm{BChl}$, and (ii) the visible region of the spectrum, which contains contribution from the bright $\left(\mathrm{S}_{2}\right)$ states of Car and the $\mathrm{Q}_{\mathrm{x}}$ states of BChls.

The elements of the exciton Hamiltonian were computed using an hybrid QM/MM model to account for the effect of the protein environment. In particular, we used two different levels of QM theory, i.e. TimeDependent Density Functional Theory (TDDFT) with the CAM-B3LYP functional, ${ }^{?}$ and the RASSCF/RASPT2 methodology ? ?, with different Active Spaces (AS) and State Average (SA) settings in the two different regions of the spectrum (See Section S5 of the Supporting Information). The TD-DFT based calculations were performed within both an electrostatic (MM) and a polarizable embed- ding (MMPol). In the latter model, the environment is described as a set of atomic point charges and isotropic atomic polarizabilities? The RAS based calculations were performed only within the electrostatic embedding of fixed point charges. Higashi et al. showed CAMB3LYP to be inaccurate for the description of solvatochromic shifts when coupled with an electrostatic embedding, and proposed to tune the range-separation parameter. ? ? However, the exciton structure obtained in Ref. ? with CAM-B3LYP/MMPol well reproduced the exciton splitting of the B850 ring, therefore CAM-B3LYP is appropriate for describing excitation with a polarizable embedding. Further details on the quantum chemical calculations are reported in Section S2 of the Supporting Information.

The electronic couplings in the TDDFT/MMPol scheme were computed as the Coulomb interaction between the transition densities of the interacting moieties.? Note that the polarizable environment adds an explicit screening term to the Coulomb cou-

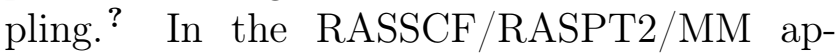
proach, the Coulomb couplings were computed with the transition density cube (TDC) $\operatorname{method}^{?}$, where no explicit screening is included. It has been shown, both theoretically $^{\text {? }}$ and experimentally, ${ }^{\text {? }}$ that the nearestneighbour $\mathrm{Q}_{\mathrm{y}}$ couplings in the $\mathrm{B} 850$ ring reduce with increasing temperature; for this reason, in order to reproduce the room-temperature properties, we used the exciton Hamiltonian calculated by some of the present authors along a classical molecular dynamics (MD) trajectory.

The full set of excitonic parameters (site energies and couplings) used is reported in sections S6 and S7 of the Supporting Information.

In order to describe exciton-phonon couplings, the spectral densities (SD) of the different transitions were modeled as a sum of one overdamped Brownian oscillator ${ }^{?}$ and $M$ discrete contributions. The discrete part of the $\mathrm{SD}$, which accounts for the intra-molecular vibrations of the various molecules, was determined from a normal mode analyisis ${ }^{\text {? }}$ of the pigments in the crystal structure, and typical 
values $^{?}$ were adapted for the continuous part. Different spectral densities were explicitly used for the $\mathrm{Q}_{\mathrm{y}}$ transitions of $\mathrm{B} 850 \alpha$, B850 $\beta$ and B800 molecules, but we used an average spectral density for all $\mathrm{Q}_{\mathrm{x}}$ transitions. All details and the used parameters are reported in Section S4 of the Supporting Information.

To describe spectra and dynamics within the $\mathrm{Q}_{\mathrm{y}}$ region, we employed the disordered exciton model with both standard Redfield (sR) and modified Redfield (mR) theories. ? ? This choice is natural if one considers the strongly coupled B850 pigments; moreover, mR theory was compared against non-perturbative hierarchical equations for the B800 ring, and performed well in reproducing the linear spectra. ? Spectra and dynamics within the Car- $Q_{x}$ region were described by Förster theory, taking into account the presence of static disorder by averaging the optical properties on a Gaussian distribution of site energies. ${ }^{\text {? The choice of }}$ Förster theory is dictated by the large reorganization energy of the Cars and by the site energy gap between Cars and $\mathrm{Q}_{\mathrm{x}}$ states. Further details about the choice of the exciton dynamics theory are reported in Section S3 of the Supporting Information.

2DES Pump-Probe spectra were simulated within the supermolecule or Sum Over States (SOS) approach, ? ? using site energies, couplings and transition dipole moments either from TD-DFT or RAS based computations, and accounting for different experimental setups in the different spectral regions investigated. We adopted a spectral line shape model able to incorporate slow and fast bath dynamics, together with exciton transport between the chromophores.? ? The 2D signals, which depend parametrically on the three controlled excitation-pulse time delays $t_{1}, t_{2}$ and $t_{3}$, were displayed in the frequency domain performing a $2 \mathrm{D}$ Fourier transform along $t_{1}$ and $t_{3}$, while keeping the waiting time (or population time) $t_{2}$ fixed. Unless otherwise specified, the 2DES maps are calculated at $t_{2}=0$. We considered only those double excitations that are associated with combinations of electronic states in the single-exciton manifold, i.e., all the other higher lying states were not included in the sim- ulation of the maps. We further refined the spectra by adding proper pulse envelopes and polarizations (See Section S3 of the Supporting Information).

All TD-DFT and RAS calculations as well as all spectra simulations have been performed using an in-house modified version of Gaussian G09 ${ }^{?}$, MOLCAS $8.1^{?}$ and Spectron $2.7^{?}$, respectively.

\section{Results and Discussion}

For clarity's sake, the presentation of the results is separated in two sections, one referring to the $\mathrm{Q}_{\mathrm{y}}$ region for which standard and modified Redfield theories were used, and the other focused on the the Car- $Q_{x}$ region, for which the Förster theory was employed.

\section{1 $\mathrm{Q}_{\mathrm{y}}$ region}

The exciton Hamiltonians for the $\mathrm{Q}_{\mathrm{y}}$ region calculated with alternative strategies (namely RASSCF/RASPT2/MM, TD-DFT/MM, and TD-DFT/MMPol) are detailed in Table S4 of the Supporting Information. The absolute values of the site energies obtained on the crystal structure with the two different QM levels (RASSCF/RASPT2 and TD-DFT) are generally quite similar (within $600 \mathrm{~cm}^{-1}$ ) when the same electrostatic embedding model is used (MM). On the contrary, the introduction of a more refined polarizable embedding (MMPol) leads to a significant red-shift, due to the inductive effects of the protein residues surrounding the pigments. ' Some differences between the QM levels are instead found in the relative ordering of the site energies for the different BChls: in particular, TD-DFT models give $\mathrm{B} 800$ as the most blue-shifted, while in the RASSCF/RASPT2 calculations B800 is found between B850 $\alpha$ and $\beta$. This different behavior, which is not due to the embedding model, as both TD-DFT/MM and TDF-DFT/MMPol give the same trend, is related to the different electronic description delivered by the two QM levels at slightly different internal geometries of the BChls. 
The specific embedding model has a significant effect on the couplings: for example, the inverted behavior and the different magnitude of the $V_{\alpha \beta}^{1}$ and the $V_{\alpha \beta}^{2}$ coupling values (Table S4) in the two different levels of QM theory can safely be attributed to the screening ef-

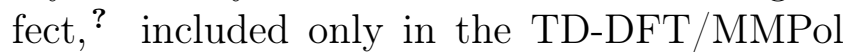
calculations through the polarizable embedding. As expected, this screening plays a significant role in determining the coupling between $\mathrm{BChls}$ within the $\mathrm{B} 850$ ring where the $\mathrm{BChl}$ are closely packed.

By using the three different Hamiltonians, the linear spectra have been generated as described in Methods Section. The results are reported in Figure ??.

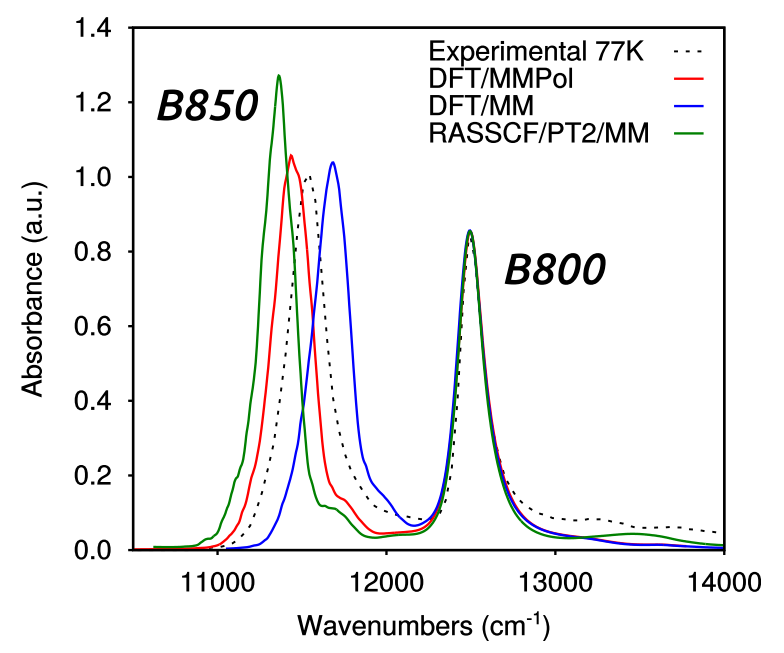

Figure 2: Comparison of linear absorption spectra of the $\mathrm{Q}_{\mathrm{y}}$ region computed at $77 \mathrm{~K}$, using modified Redfield theory, at different levels of QM theory and/or embedding models. The experimental spectrum is also reported (dashed line). The calculated spectra have been shifted to match the experimental peak at $800 \mathrm{~nm}$. The TD-DFT/MMPol, TD-DFT/MM and RASSCF/RASPT2/MM spectra have been shifted by 1280,550 , and $120 \mathrm{~cm}^{-1}$, respectively.

Due to differences in the absolute values of the site energies, the computed spectra shall be shifted individually by a different amount, in order to be compared with actual experimental data. As expected, RASSCF/RASPT2 level of QM description gives a more accurate estimation of the site energies, and the corre- sponding spectrum requires only a small (120 $\mathrm{cm}^{-1}$ ) shift to the blue to match the experimental B800 peak. A much larger blue-shift is necessary at TD-DFT level. However, in spite of the larger offset in the absolute energies, the TD-DFT/MMPol approach better reproduces the band splitting between the two peaks at 800 and $850 \mathrm{~nm}$, due to a more balanced description of site energy differences and couplings. The latter, in fact, correctly accounts for the possible screening effects as a polarizable embedding (MMPol) has been used. For this reason, we employed TD-DFT/MMPol data to model the 2DES maps.

In Figure ?? we report the simulated maps at zero population time and temperatures $77 \mathrm{~K}$ (d) and $300 \mathrm{~K}(e)$, along with the experimental map $(f)$ of the bacterium Rps. Palustris $^{?}$ in the same spectral region, acquired at zero population time and $300 \mathrm{~K}$. The $77 \mathrm{~K} 2 \mathrm{DES}$ map was computed using the TD-DFT/MMPol Hamiltonian calculated on the crystal structure, whereas for the $300 \mathrm{~K}$ map we employed the MD Hamiltonian from Ref. ? . The pulse shapes were designed in order to resemble the experimental pulse envelopes (approximately the same overlap with the various bands as it appears in the experimental spectrum).

The simulated maps at the two temperatures show a similar positioning of peaks and crosspeaks, but, as expected, the $77 \mathrm{~K}$ map is dominated by the site energy static disorder (peaks extended along the diagonal), while $300 \mathrm{~K}$ map shows much broader peaks. We observe a considerable similarity between simulated and experimental maps at $300 \mathrm{~K}$ especially in the relative position, shape and intensity of the positive and negative B850 signals. To this respect, it has been shown by Fleming et al. that the positioning of these positive and negative B850 related features can be used as a coarse-grained probe for structural insights on the studied system. Symmetrically, when the structure is already known (such as for the LH2 of Rps. Acidophila), the correct reproduction of positioning, shapes and relative intensities of these features supplies an indirect proof of the accuracy of both the underlying quantum chemical method and the spectra simulation proto- 

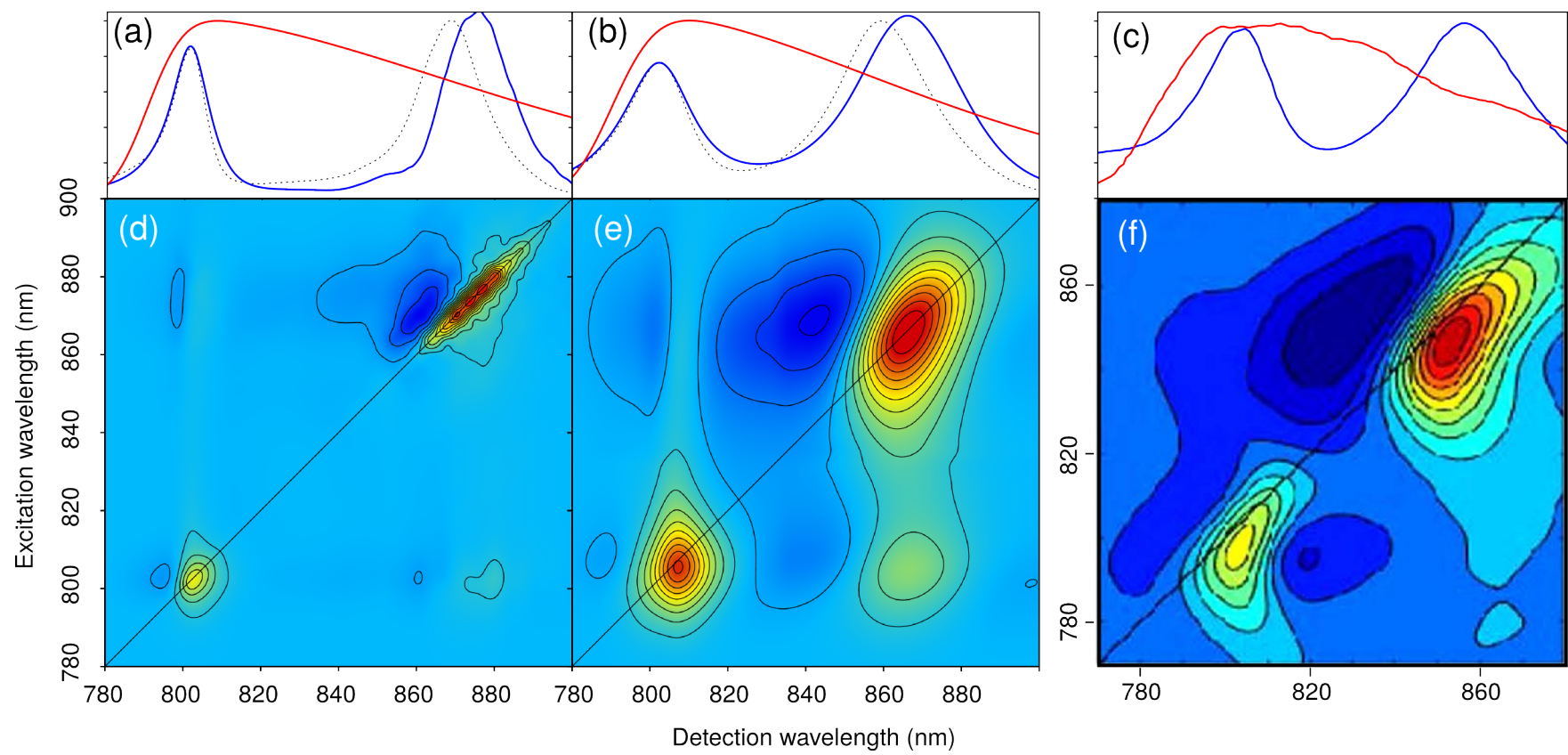

Figure 3: Comparison of 2DES pump-probe maps at zero population time. Simulated maps at $77 \mathrm{~K}(d)$ were computed for the TD-DFT/MMPol Hamiltonian. Simulated maps at 300K $(e)$ were computed for the MD Hamiltonian. ${ }^{?}$ All simulated maps were shifted to match the experimental peak at $800 \mathrm{~nm}$. The experimental map at 300K $(f)$ refers to the LH2 of Rps. Palustris. ${ }^{?}$ The corresponding linear absorptions (blue) and pulse shapes (red), along with the relative experimental linear spectra (dashed line), ${ }^{?}$ are reported in $(a),(b)$ and $(c)$, respectively. Simulated maps are normalized between their minimum and maximum values. By convention, bleach and stimulated emission contributions appear as positive (red) signals, excited state absorption appear as negative (blue) peaks. The experimental map has been adapted from Ref. ? .

cols used. With regard to B800 signals, strong excited state absorption (ESA) signals on both sides of the main diagonal are present in the experiment, whereas the same signals appear to be weaker in the computed maps. We remark that our simulated ESA signals only arise from combinations of electronic states in the singleexciton manifold; thus, we did not include ESA signals related to the excitation of the higher lying Soret bands from the $\mathrm{Q}_{\mathrm{y}}$ transition. As the frequency of such excitations is compatible with these negative ESA on both sides of the positive bleaching, it is possible that $\mathrm{Q}_{\mathrm{y}} \rightarrow$ Soret excitations contribute to the negative signals around the diagonal B800 peak. Furthermore, it should also be noted that calculations and experiments refer to different (even though similar) bacteria.

A good agreement between experimental and theoretical maps can be found also in the offdiagonal regions, with similar positive and negative features in both sides of the maps. The presence of these off-diagonal signals in the spectra reflects the weak but still non-negligible coupling between the excitons of the two rings, which is accurately taken into account in our QM/MMPol model. In fact, these off-diagonal signal are present also in the rephasing maps measured by Harel and Engel for the LH2 of $R b$. Sphaeroides. ' In order to ease the comparison, the rephasing 2DES map (absolute value) is reported in Figure S11 of the Supporting Information.

Remarkably, even if all the relevant excited states are properly included in the 2DES simulations, one should also consider the proper shape of excitation and detection pulses, as implemented here. This refinement is mandatory if, for example, one is interested in comparing the intensities of B800 and B850 bands in simulated and experimentally recorded 2D maps.

The downhill relaxation rates among the $\mathrm{Q}_{\mathrm{y}}$ excitons of LH2 were analyzed for over 10000 
realizations of static disorder site energy distributions, at $77 \mathrm{~K}$ and room temperature. For each realization of the disorder, the exciton states were assigned to the B800(B850) ring on the basis of the participation of the B800(B850) pigments to the exciton state. The average of 10000 realizations is shown in Table ?? as a relaxation time. We show the average of all $\mathrm{B} 800 \rightarrow \mathrm{B} 800, \mathrm{~B} 800 \rightarrow \mathrm{B} 850$, and $\mathrm{B} 850 \rightarrow$ B850 transfer rates, along with the average of the maximal rates taken within every realization.

Calculated B800 $\rightarrow$ B850 transfer times are around $0.6-1 \mathrm{ps}$, in line with several experimental findings. ? ? ? ? ? The rates depend on temperature, with the $\mathrm{mR} 300 \mathrm{~K}$ rates being $66 \%$ faster than the $77 \mathrm{~K}$ rates. This is in agreement with the temperature dependence of the B800 $\rightarrow$ B850 energy transfer in Rps. Acidophila determined by pump-probe spectroscopy, where the $300 \mathrm{~K}$ rate is $\sim 60 \%$ faster than the $77 \mathrm{~K}$ rate. ' Similar measurements on the LH2 of $R b$. Spheroides show a similar behaviour for the inter-ring transfer times.

The intra-B800 relaxation rates are much harder to determine experimentally; anisotropy decay spectroscopy at different temperatures on Rps. Acidophila shows that the intra-B800 transfer occurs on a 400-800 fs time scale at room temperature, ${ }^{?}$ in agreement with threepulse photon echo studies. ' Our intra-B800 $300 \mathrm{~K}$ transfer rates are much faster, indicating the limits of modified Redfield theory in describing the intra-B800 dynamics. Conversely, at $77 \mathrm{~K}$, the rates are much closer to the experi-

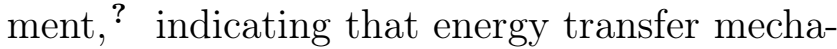
nism is more similar to excitonic at lower temperature.

The results also show a weak dependence of the transfer rates on the excitation transfer theory adopted. The main difference between sR and $\mathrm{mR}$ theories is that in $\mathrm{mR}$ multiple quanta of energy can be exchanged between excitons and phonons, even though sR exciton transfer rates can be larger than $\mathrm{mR}$ rates. ? Modified Redfield reduces to standard Redfield when the site energies approach degeneracy. ? ? Our results point to a small influence of multi-phonon relaxation, for the average relaxation pathways, within our combination of exciton Hamiltonian and energetic fluctuations.

Table 1: Average transfer times (in fs) of the LH2 ring. Numbers in parentheses refer to the average of maximum rates taken within every realization. Different columns refer to different temperatures. The $300 \mathrm{~K}$ rates were computed on the MD Hamiltonian. ? Only downhill rates have been considered in this analysis.

\begin{tabular}{|c|c|c|}
\hline & $77 \mathrm{~K}$ & $300 \mathrm{~K}$ \\
\hline \multicolumn{3}{|c|}{ Modified Redfield } \\
\hline $\mathrm{B} 800 \rightarrow \mathrm{B} 800$ & $321(81)$ & $196(60)$ \\
\hline $\mathrm{B} 850 \rightarrow \mathrm{B} 850$ & $244(36)$ & $137(25)$ \\
\hline $\mathrm{B} 800 \rightarrow \mathrm{B} 850$ & $1045(85)$ & $630(79)$ \\
\hline \multicolumn{3}{|c|}{ Redfield } \\
\hline $\mathrm{B} 800 \rightarrow \mathrm{B} 800$ & $317(100)$ & $168(64)$ \\
\hline $\mathrm{B} 850 \rightarrow \mathrm{B} 850$ & $244(26)$ & $142(19)$ \\
\hline $\mathrm{B} 800 \rightarrow \mathrm{B} 850$ & $1032(79)$ & $670(65)$ \\
\hline
\end{tabular}

Further insight in the rate analysis can be obtained by investigating the energy dependence of the relaxation rates. We report in Figure ?? two-dimensional histogram plots of the exciton relaxation rate vs. exciton energy at $77 \mathrm{~K}$. Notably, the exciton relaxation rates strongly depend on the exciton energy. In the low-energy B850 region $\left(<12400 \mathrm{~cm}^{-1}\right)$ the exciton relaxation rate rises with the energy; a region with slow relaxation rates can be found around 12500 $\mathrm{cm}^{-1}$, due to the low end of the B800 band. At the blue edge of the B850 band we notice excitons with a very fast relaxation rate (tens of femtoseconds). We also compared the relaxation rates of the full LH2 system with a Hamiltonian where the B800 ring is decoupled from the B850 ring. The comparison with the artificially decoupled system shows that coupling to the B850 states shortens the lifetime (enhances the relaxation rate) of the B800 states. Finally, as the B850 states in this energy region are optically dark, their lifetime can only be measured if these states are mixed with the B800 states. In the B800 region the exciton lifetime generally decreases at higher energies. This is in agreement with polarized pump-probe measurements 
and rate calculations on the LH2 of Rs. Molischianum at $77 \mathrm{~K} .{ }^{?}$ Although our computed exciton lifetimes are shorter than the measured polarization relaxation times, it is important to notice that exciton relaxation rates also include processes that may not change the polarization of the transition.?

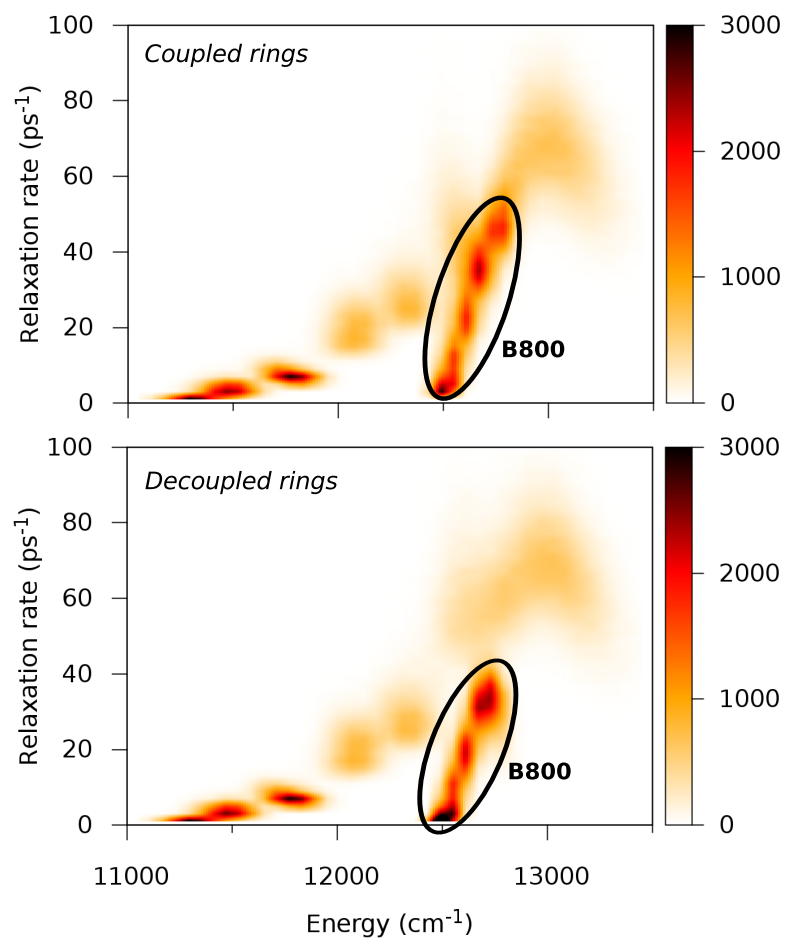

Figure 4: Two-dimensional histogram plots of exciton relaxation rates versus exciton energies ( $\mathrm{mR}$ theory using the TD-DFT/MMPol exciton Hamiltonian at $77 \mathrm{~K}$ ). The histograms show the distribution of energy and relaxation rates over 10000 realizations of the site energy static disorder. The color range indicates the number of occurrences. The contribution of the B800 states is highlighted with a circle. Top: complete Hamiltonian with coupled rings; bottom: Hamiltonian with the B800 ring decoupled from the B850 ring.

A detailed statistics of delocalization length, dipole strength, and transfer rates in the three lowest exciton states of LH2, which belong to the B850 ring, is reported in Figure S10 of the Supporting Information.

\subsection{Carotenoid- $\mathrm{Q}_{\mathrm{x}}$ region}

The Car-Qx linear spectra at both $77 \mathrm{~K}$ and 300K were simulated as described in the Methods Section using the site energies and couplings reported in Table S6 of the SI. Due to the multi-excitation nature of the Car states, only the RASSCF/RASPT2/MM level has been used for this spectral region.

The results at both temperatures, together with the relative experimental spectra, are reported in Figure ??.

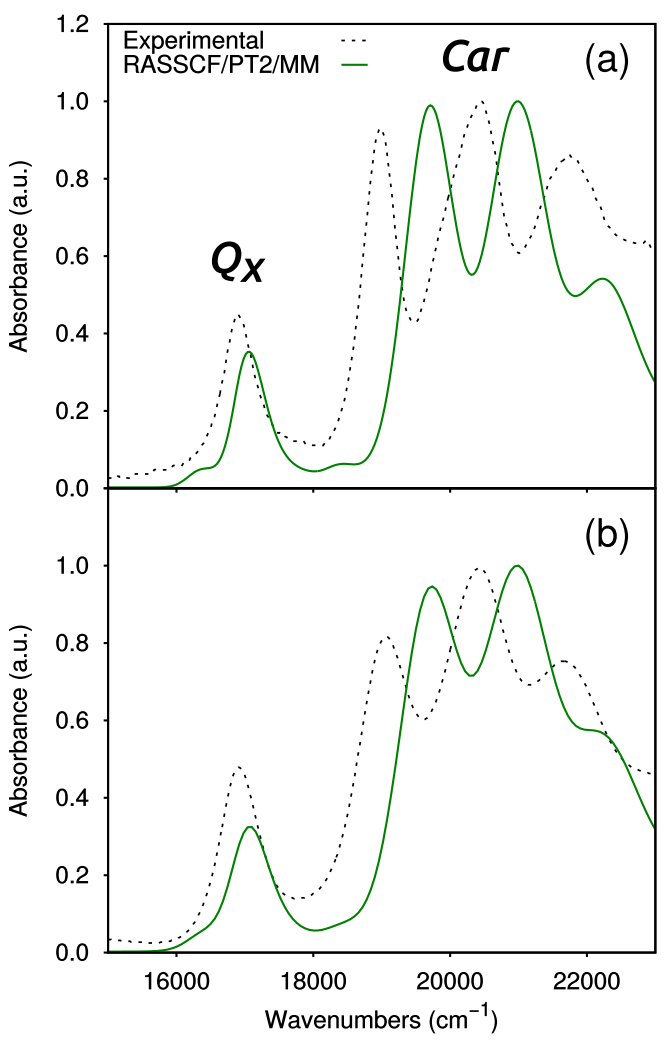

Figure 5: Linear absorption spectra for the Car- $\mathrm{Q}_{\mathrm{x}}$ region computed at $77 \mathrm{~K}(a)$ and $300 \mathrm{~K}$ (b) at the RASSCF/RASPT2/MM level of theory. The experimental spectra are also reported (dashed line), taken from Ref. ? . All the spectra have been normalized with respect to the maximum peak. Calculated spectra have not been shifted.

The deviations in the relative intensities between experimental and calculated vibronic bands can origin from different factors. However, the slight offset in the relative intensities of the Car vibronic peak seems to point to a possible underestimation of the Huang-Rhys fac- 
tors for the $\mathrm{S}_{2}$ state, which leads to a general overestimation of the $0-0$ transition intensity.

Differently to the $\mathrm{Q}_{\mathrm{y}}$ region, available experimental 2D maps in the Car- $Q_{x}$ region refer exactly to the same photosynthetic organism, namely the Rps. Acidophila.

In Figure ??, we interpret the origin of experimental signals by artificially disentangling the Car- and BChl-related regions. For such an analysis, we refer to the extrapolated map (corresponding to $t_{2} \rightarrow 0$ ) obtained by Ostroumov

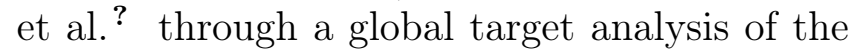
measured 2D data.

From the target analysis, the three diagonal contributions in the extrapolated map were assigned to ground state bleaching (and SE) signals from the Car $\mathrm{S}_{2}$ (full triangle of ??), BChl $\mathrm{Q}_{\mathrm{x}}$ states (empty triangle), and an additional dark state of Car located in between the $S_{2}$ and $\mathrm{Q}_{\mathrm{x}}$ transitions, the so called $X$ state (empty circle). The strong below-diagonal cross-peak (full diamond) was attributed to an ultrafast $\mathrm{S}_{2} \rightarrow X$ energy transfer, whereas for the abovediagonal cross-peak (empty square) the origin was associated to a ground state bleaching arising from mixed excitation of coupled $Q_{x}$ and Car $X$ states.

Our calculated map of the Car region (Figure ?? a) shows signals originating from the $S_{2}$ state and the related vibrations. On the diagonal, the bleaching is the signal with the highest intensity, while the off-diagonal sequence of peaks probing at larger detection wavelengths has to be assigned to the $S_{2}$ stimulated emission. We also note that the broad $S_{2}$ signals have significant contributions along the diagonal towards larger wavelengths.

The analysis of the $\mathrm{Q}_{\mathrm{x}}$ signals in the calculated BChls map (Figure ??c) shows that the positive elongations of the experimental $\mathrm{Q}_{\mathrm{x}}$ transition at smaller excitation and detection wavelengths with respect to the bleaching, originate from the vibronic progression of the $Q_{x}$ (the empty square and diamond in the figure). This vibronic progression is also responsible of a weaker diagonal signal around $550 \mathrm{~nm}$ (the empty circle in the figure).

The calculated spectra for the separated Car and BChl contributions explain the main fea- tures of the extrapolated map in terms of the Car bright $\left(S_{2}\right)$ state combined with the $\mathrm{Q}_{\mathrm{x}}$ vibronic progression of BChls. We must note, however, that the present theoretical analysis is limited to early $t_{2}$ population times; therefore, we cannot exploit the time-dependent evolution of the signals to definitely check the validity of the suggested interpretation. Nevertheless, an interesting result seems to come out clearly: the signals on and below the diagonal at the $560 \mathrm{~nm}$ excitation wavelength cannot be unambiguously assigned to Car or BChl due to overlaps of the $S_{2}$ and $\mathrm{Q}_{\mathrm{x}}$ related signatures. Instead, the off-diagonal peak above the diagonal (indicated with the empty square in Figure ??b) is not significantly affected by any $S_{2}$-related signal. It should be added that the oscillations of the off-diagonal peak have been investigated using a global target analysis of the to absorptive 2D signals. ' The resulting vibration-associated spectra (2DVAS) have been compared to those of the isolated Car and the observed similarity has been used to confirm the Car origin of the signal. However, our calculations show that only the $\mathrm{Q}_{\mathrm{x}}$ vibronic progression contribute to this spectral feature, while $S_{2}$ is completely inactive. To unify the theoretical and the experimental findings it is necessary to invoke the presence of a Car dark state, either an additional electronic state (the $X$ state) or a very hot vibrational level of the low-lying $S_{1}$ state. The RASSCF/RASPT2 calculations give an electronic state with the same symmetry of $S_{2}$ but laying well above $S_{2}$; however, previous multireference DFT calculations? have shown that the relative position of the electronic states in Cars strongly depend on the geometry through variations in the bond length alternation. It is thus possible that geometrical fluctuations and/or distortions can bring the $X$ state down below the bright one.

Once analyzed the contributions of the two pigments separately, in Figure ?? we report a comparison between the computed Car- $\mathrm{Q}_{\mathrm{x}}$ coupled map and the experimental spectrum at early $t_{2}$ time.

At this short time, the Car region is expected to be dominated by the $S_{2}$ state and higherenergy states reached from it. However, some 

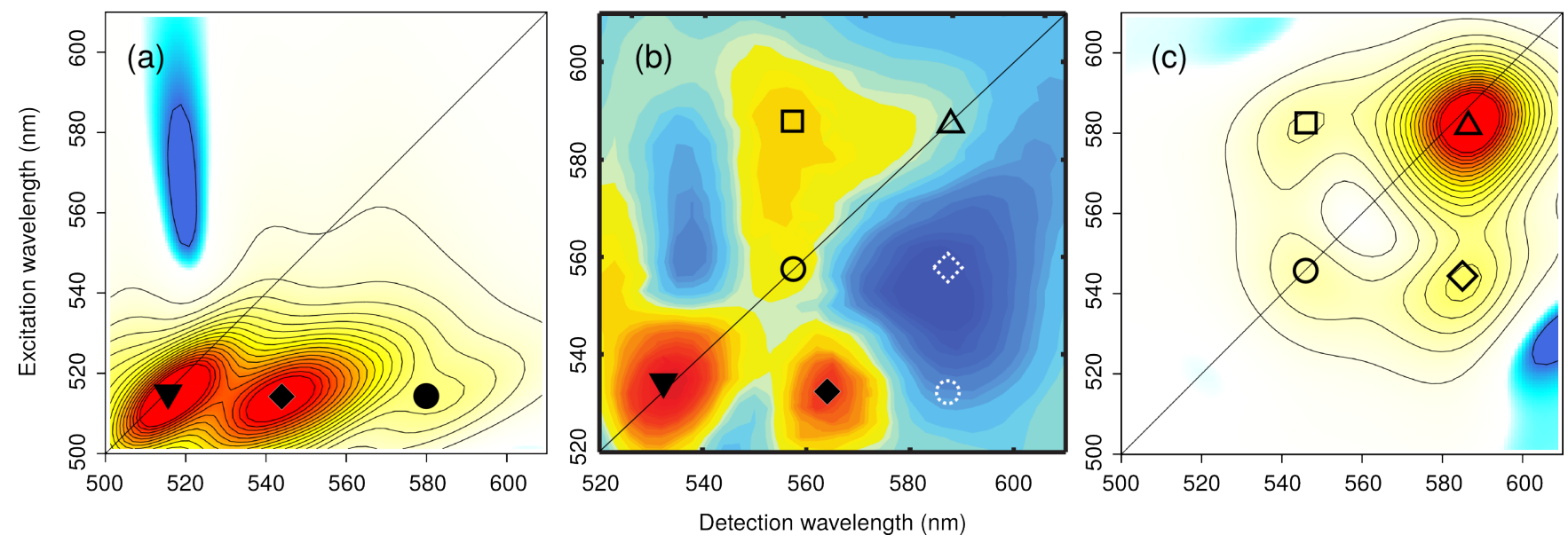

Figure 6: Calculated 2DES pump-probe maps at $t_{2}=0$ obtained by separately computing Cars' $(a)$ and BChls' $(c)$ contributions. (b) Short $t_{2}$ component of the $2 \mathrm{D}$ evolutionary-associated spectra obtained through a global target analyis of the experimental $2 \mathrm{D}$ data. ${ }^{?}$ The effect of the pulse shape has been taken into account in the simulated maps. All the maps are truncated at $75 \%$ intensity. By convention bleach and stimulated emission contributions appear as positive (red) signals, excited state absorption appear as negative (blue) peaks. Full (referring to Cars) and empty (referring to BChls) geometric symbols are used to indicate correlations between calculated and measured signals.

features due to the dark $S_{1}$ state cannot be excluded, as assuming an internal conversion process 100-150 fs long, $S_{1}$ can still be populated from $S_{2}$. Indeed, we note that the negative signals at the red side of the $S_{2}$ bleaching, absent in the $t_{2} \rightarrow 0$ extrapolated map, and rapidly increasing at larger $t_{2}$ times, are likely due to the ESA from both $S_{2}$ and $S_{1}$. As in our calculations the internal conversion to $S_{1}$ is not allowed and both $S_{1 \rightarrow n}$ and $S_{2 \rightarrow n}$ ESA signals are not included, these negative signals are completely absent. Because, these negative signals lower the intensity and modify the shape of the positive $S_{2}$ SE, a much more intense and less redshifted signal is present in the calculated map (see Figure ??). We also observe that the negative signal at increasing excitation wavelengths also contains pure $S_{2}$ contributions, since a similar feature also appears in the Car-only simulated map (Figure ??a).

As previously commented for the linear spectra, the relative intensity of the $\mathrm{Q}_{\mathrm{x}}$ band with respect to the Car $S_{2}$ is weaker in the computed maps compared to the experiment. This difference is even more pronounced in the 2D (Figure ??d) due to the multiple interactions between light and the transition dipole moments of the molecules, which characterize this technique. Moreover, the calculated gap between $\mathrm{S}_{2}$ and $\mathrm{Q}_{\mathrm{x}}$ states is slightly too large leading to a too small spectral overlap between these two signals. To check the effects of these deviations, we have recalculated the $2 \mathrm{D}$ map by introducing two corrections: (i) we have introduced a scaling of the $\mathrm{Q}_{\mathrm{x}}$ transition dipoles so to match the relative absorption intensities of the experimental linear spectrum and (ii) we have separately red-shifted the Car $S_{2}$ and the BChl $\mathrm{Q}_{\mathrm{x}}$ site energies so to match the corresponding measured peaks in the linear spectrum (see the linear spectra reported in Figure ??).

The comparison between the corrected (Figure ??e) and the original calculated 2D maps shows that the main features are fully preserved, telling us that the method used provides a robust description. This is further confirmed by the analysis of the calculated rates of $S_{2} \rightarrow \mathrm{Q}_{\mathrm{x}}$ energy transfer, obtained applying the Förster theory. These transfers can be separated into three channels, that is, from

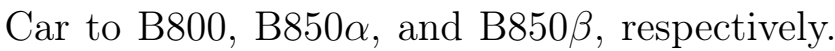
The fastest energy transfer occurs to the $\mathrm{Q}_{\mathrm{x}}$ state of BChl B850 $\alpha$, which is the most coupled to $S_{2}\left(175 \mathrm{~cm}^{-1}\right.$ from our calculations $)$ ? 


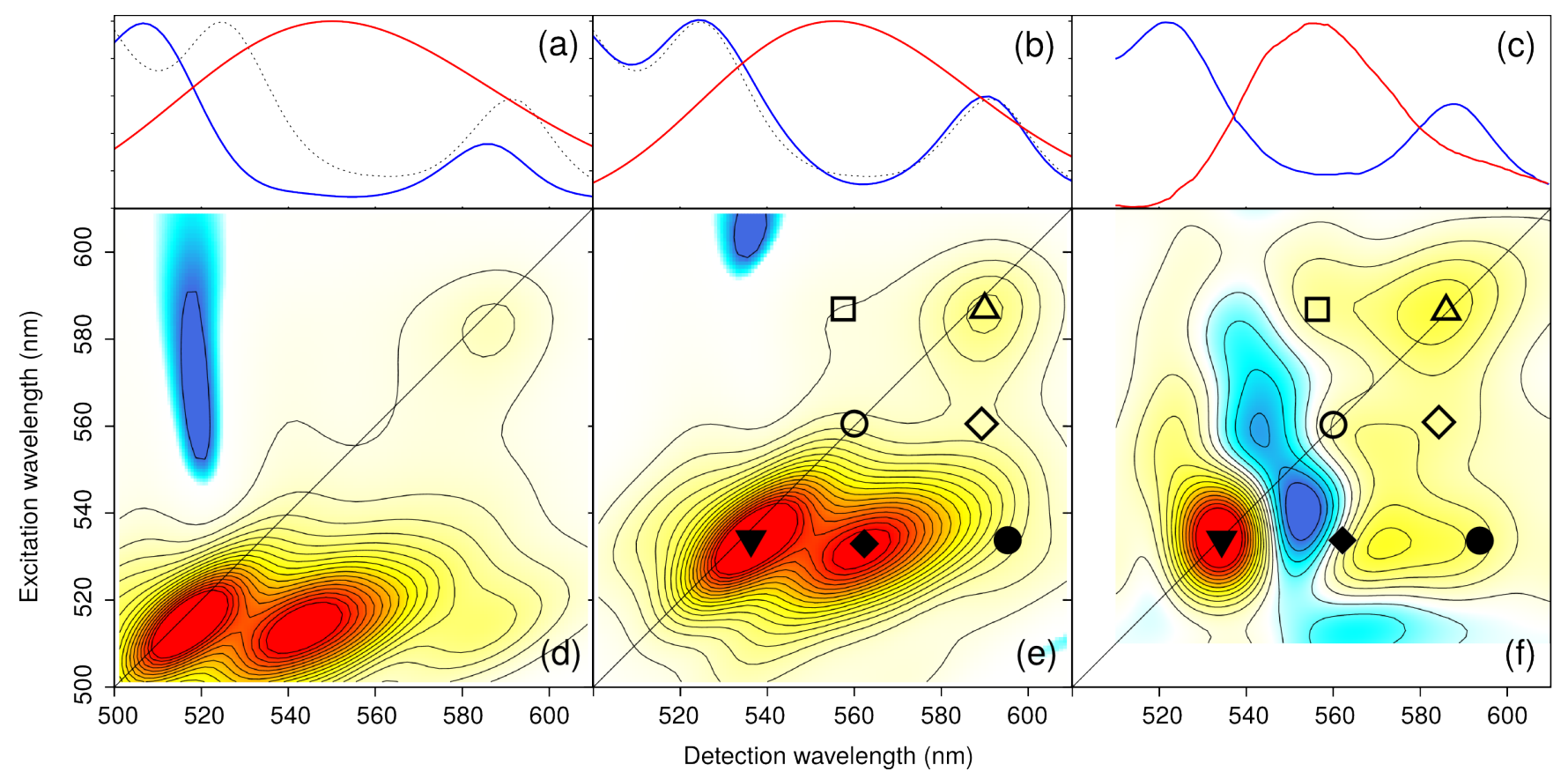

Figure 7: Comparison of simulated $(d, e)$ and experimental ${ }^{?} \quad(f)$ 2DES pump-probe maps of Rps. Acidophila at $300 \mathrm{~K}$ and waiting time $t_{2}=22 \mathrm{fs}$. The corresponding linear absorptions (blue) and pulse shapes (red), along with the relative experimental linear spectra (dashed line), ${ }^{?}$ are reported in $(a),(b)$ and $(c)$, respectively. In the calculated maps the pulse shape was adapted to have the same overlap between pulses and absorption bands as the one reported in the experiments. Panel (e) refers to a calculated map where Car $S_{2}$ site energies and $\mathrm{Q}_{\mathrm{x}}$ transition dipoles have been scaled to match the position and the intensities measured in the linear spectrum. All the maps are truncated at $75 \%$ intensity. By convention, bleach and stimulated emission contributions appear as positive (red) signals, excited state absorption appears as negative (blue) peaks. The geometric symbols represent the same peaks as in Figure ??.

Its EET rate is predicted to be around 140 $\mathrm{fs}^{-1}$ (or $110 \mathrm{fs}^{-1}$ if we use the corrected energies). We predict slower EET rates to B800 and B850 $\beta$, around $1 \mathrm{ps}^{-1}$. The total $S_{2}$ depopulation time for EET to $Q_{\mathrm{x}}$ states is $\sim 120$ fs (or 90 fs with the corrected energies). Assuming that the $S_{2} \rightarrow S_{1}$ internal conversion rate of the Car is $130 \mathrm{fs}^{-1},{ }^{?}$ ? we estimate a $59 \%$ efficiency of energy transfer from the Car to the $\mathrm{Q}_{\mathrm{x}}$ states, and a $48 \%$ efficiency of EET to B $850 \alpha$ alone. These results are in good agreement with several experiments, ? ${ }^{?}$ and in particular with the rates estimated from fluorescence upconversion and coupling calculations. ${ }^{\text {? }}$ Our calculations predict that the $\mathrm{Q}_{\mathrm{x}}$ state of $\mathrm{B} 850 \alpha$ is the main energy acceptor from $S_{2}$.

\section{Conclusion}

Many examples of quantum chemistry based approches to describe LH complexes through excitonic models have been reported in the literature (for a recent review see Ref. ? ). However, the majority of these theoretical investigations concerned linear absorption spectra with very rare extensions to 2DES spec-

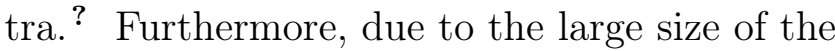
multichromophoric aggregate of LH complexes, and the very heterogeneous composition of the embedding, rather approximated QM methods (mostly of semiempirical nature) and/or simplified descriptions of the environment have been generally used. Indeed, the application of accurate quantum mechanical methods have been mostly limited to benchmark studies on the electronic properties of single pigments.

Here, for the first time, the simulation of lin- 
ear and 2DES spectra of LH2 over the whole Car-Qx plus $Q_{y}$ VIS-NIR region has been presented by integrating quantum-chemistry and electron-phonon exciton models. Single and multireference QM methods have been combined with electrostatic and polarizable classical embeddings to properly account for the effect of the protein in all the steps of the simulation. On the one hand, TD-DFT/MMPol computations have been proved to achieve a refined description of the effect of the environment in the $\mathrm{Q}_{\mathrm{y}}$ region via a precise reproduction of the B800-B850 bands splitting. On the other hand, the multiconfigurational multireference RASSCF/RASPT2/MM approach provides an accurate description of the Car- $\mathrm{Q}_{\mathrm{x}}$ region. All these data were used as input parameters for the construction of exciton Hamiltonians able to describe the manifold of states of the LH2 complex.

In particular, we have shown that, in addition to the molecular components and the accurate description of the environment effects, the modeling of 2D maps must include the pulse envelopes in order to achieve a reliable and complete comparison with the experiments. Most importantly, the possibility of artificially switching on and off interactions among the different components of the multichromophoric systems, provides a unique tool for interpreting the origin of the different signals which overlap in the highly congested experimental spectrum. This analysis has shown its effectiveness in the Car- $\mathrm{Q}_{\mathrm{x}}$ region where we have given a possible interpretation of the main features of the very-short time 2DES spectrum in terms of the bright $S_{2}$ and $\mathrm{Q}_{\mathrm{x}}$ vibronic progression of Car and BChl, respectively. By combining these results with those obtained from a global target analysis applied to 2DES data, ${ }^{?}$ we have reinforced the hypothesis that a contribution coming from a dark state of the carotenoid lying in between the $S_{2}$ and the $\mathrm{Q}_{\mathrm{x}}$ is present; if this is an additional electronic state (the previously suggested $X$ state) or an high-energy vibrational level of the $S_{1}$ is still an open question that calls for further investigation.

Here, we have shown that the way to be followed is the integration of multiscale models and experiments: only by combining the "independent" results from accurate quantum chemical based methods and detailed 2DES experiments, the complex network of energy transfer routes in LH2 (and in other LH systems) can be definitely revealed. To do that, however, two important improvements are still needed in the theoretical strategy here presented. The ESA signals of Cars, in particular those from $S_{1}$, which become increasingly important for longer waiting time $t_{2}$, have to be explicitly included to follow the system evolution and simulate time resolved 2D maps. Moreover, a detailed account of possible geometrical distortions of the pigments (especially the Cars) due to temperature-dependent fluctuations of the protein is necessary, as this mechanism could affect the relative energy and the nature of the excited states involved in the excitons and in the energy transfer processes.

Supporting Information Available: Details on the preparation of the structure. Excitonic parameters for both the $\mathrm{Q}_{\mathrm{y}}$ and Car- $\mathrm{Q}_{\mathrm{x}}$ regions. Spectral densities of the exciton-phonon coupling. Details of the RASSCF/RASPT2 calculations. Analysis of the relaxation rates in the $\mathrm{Q}_{\mathrm{y}}$ region. This material is available free of charge via the Internet at http://pubs.acs.org/.

Acknowledgement The authors thank Gregory Scholes and Evgeny Ostroumov for the short-time 2DES map in the Car- $\mathrm{Q}_{\mathrm{x}}$ region and for their valuable comments on the comparison of calculated and experimental maps. B.M., S.J. and L.C. acknowledge support by the European Research Council Starting Grant ENLIGHT (ERC-2011-StG No. 277755). M.G. acknowledges support by the European Research Council Advanced Grant STRATUS (ERC-2011-AdG No.291198) and of the French Agence National de la Recherche (FEMTO2DNA, ANR-15-CE29-0010). 
Graphical TOC Entry

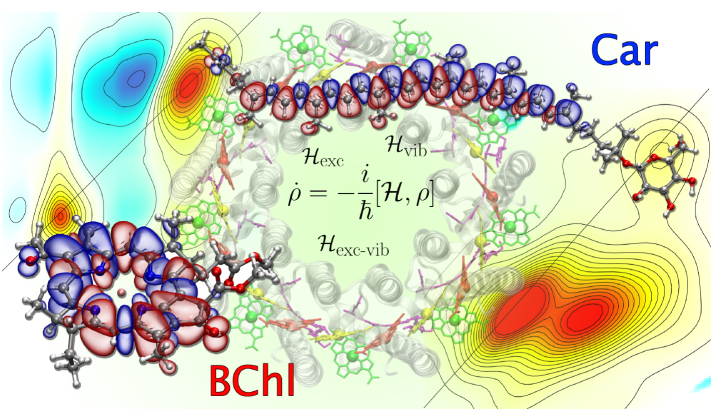

